\title{
Towards a Predictive Model for Initial Chlorine Dose in Humanitarian Emergencies
}

\author{
Hongjian $\mathrm{Wu} *$ and Caetano C. Dorea $(\mathbb{D}$ \\ Department of Civil Engineering, University of Victoria, Victoria, BC V8P 5C2, Canada; caetanodorea@uvic.ca \\ * Correspondence: jeffuvic@gmail.com
}

Received: 28 April 2020; Accepted: 22 May 2020; Published: 25 May 2020

\begin{abstract}
Free chlorination is a widely employed disinfection method in humanitarian water provision due to its many advantages. However, its effective application is hindered by the challenge in determining adequate initial doses to achieve free chlorine residuals that satisfy both health and aesthetic requirements. Current guidelines show varying recommended dosing strategies, and many do not adequately consider chlorine decay mechanisms that occur during water storage. Even though turbidity is commonly used as a criterion for deciding chlorine dose, it may not be an adequate proxy for the water quality in many cases. This paper addresses the fundamental relationships between chlorine decay kinetics and selected key water parameters (i.e., natural organic matter, water temperature, chlorine demand) by conducting chlorine decay tests in controlled conditions and in jerrycans (i.e., simulating humanitarian water treatment conditions). Chlorine decay constant from the Feben and Taras's empirical model and first order model formed linear and exponential relationships with two water parameters $\left(\mathrm{UVA}_{254}\right.$ and 30-min chlorine demand). With these relationships, the two chlorine decay models can be calibrated quickly and frequently in the field, allowing effective determination of initial chlorine dose. These two models calibrated based on the suggested water parameters from the study could predict chlorine decay in water having a main chlorine demand-inducing constituents as natural organic matter. However, they underpredicted chlorine decay in surface water with additional chlorine reactants. Further research on additional chlorine decay mechanisms is needed to expand the applicability of the models.
\end{abstract}

Keywords: chlorine decay; natural organic matter; temperature; humanitarian emergency; WASH; bucket chlorination

\section{Introduction}

Humanitarian emergency situations arise when natural or man-made hazards bring serious disruptions to a society, causing widespread human suffering, and stretch the community's coping mechanisms to a breaking point [1]. Damages to local water systems or mass migration in some cases may lead to a lack of water to meet the community's basic needs. Insufficient quantity of water for consumption and hygiene, together with poor water quality, underlies most public health problems such as transmission of diarrheal diseases during crisis situations [2]. Therefore, it is critical for humanitarian relief organizations to provide water of safe drinking quality and in adequate volume to support lives and hygiene in the community [2-4]. For humanitarian water provision, disinfection is an essential step to produce water of safe drinking quality as it aims to inactivate disease-causing microorganisms in water.

There are a variety of water disinfection methods for application in the context of a humanitarian emergency (referred to as "the field" hereinafter) such as water boiling, chlorination, ultraviolet disinfection, etc. [5]. However, free (or breakpoint) chlorination is usually the method of choice because chlorine is relatively easy to obtain, apply, measure, and it can inactivate most viral and 
bacterial pathogens in a rapid and cost-effective manner, in addition to leaving a residual protection in the water [6-8].

Despite the various advantages of free chlorination in the field, a key challenge for its effective application is finding the appropriate doses to achieve protective residual at a target storage time at the point of use [7]. Storage time is typically $24 \mathrm{~h}$, assuming water is fetched daily. Within the 24-h storage time, the free chlorine residuals (FCR) need to meet both recommended health-derived and aesthetic objectives [9]. This creates the challenge because chlorine is a strong oxidant and may react with a variety of constituents (i.e., metals, organics, ammonia, etc.) in water; hence, its concentration decays over time at a rate that depends on various factors [10]. Some of these factors include quality of the treated water, water temperature, initial chlorine dose, and/or the reaction container (details of which are discussed later).

Field conditions set humanitarian water treatment apart from conventional treatment contexts. In the latter, chlorination is achieved in engineered tanks and pipelines under relatively stable environmental conditions. In humanitarian water treatment, chlorination may take place in locations ranging from centralized water tanks to decentralized collection and storage containers (e.g., buckets, jerrycans, etc.) at the point of collection or point of use in a humanitarian context [9]. The treated water may often be subjected to elevated temperatures [11], which accelerates chemical reactions in the water, leading to higher FCR decay rates [11-13]. For FCR verifications, pool testers and several other measuring devices are often used, and the results are less consistent compared to those resulting from using a colorimeter because pool testers require operators' personal judgement [8]. The limitation of imprecise readings with the field instruments makes it challenging to obtain key information (i.e., chlorine demand, FCR decay data, etc.) Both the environmental conditions and instrumentation in humanitarian contexts add uncertainties to initial chlorine dose prediction.

To guide chlorination application in humanitarian contexts, varied international guidelines and research studies provide recommendations on the initial doses based on source water characteristics. A comparison of the recommendations is provided in Table 1.

Table 1. Chlorine dosages recommendations from several guidelines and studies.

\begin{tabular}{|c|c|c|}
\hline Guidelines/Studies & Chlorine Dose Recommendations & References \\
\hline CDC & $\begin{array}{c}1 / 8 \text { teaspoon ( } 8 \text { drops) of bleach }(5-6 \% \text { or } 8 \% \text { ) for each gallon of clear water; } \\
\text { Double value for cloudy water }\end{array}$ & [14] \\
\hline US EPA & $\begin{array}{l}\left.2 \text { drops to } \frac{1}{2} \text { teaspoon of bleach ( } 6 \% \text { or } 8.25 \%\right) \text { to certain amount of water } \\
\qquad(1 \mathrm{~L} \text { to } 8 \text { gallons) }\end{array}$ & [15] \\
\hline \multicolumn{3}{|l|}{ Exact Values } \\
\hline Lantagne & $\begin{array}{l}1.875 \mathrm{mg} / \mathrm{L} \text { free chlorine for water with turbidity }<10 \mathrm{NTU} \\
3.75 \mathrm{mg} / \mathrm{L} \text { free chlorine for water with turbidity } 10-100 \mathrm{NTU}\end{array}$ & [9] \\
\hline JHU and IFRC & $\begin{array}{l}2.50 \mathrm{mg} / \mathrm{L} \text { free chlorine, verify that there is at least } 1.00 \mathrm{mg} / \mathrm{L} \text { free chlorine } \\
\text { residual at } 30 \mathrm{~min} \text { after dosing }\end{array}$ & [16] \\
\hline
\end{tabular}

Based on the comparison of listed recommendations, there are four points to be noted. Firstly, the descriptive based guidelines are comprehensive, which allow for most people to execute. However, the bleach solution may have degraded [18], and no information was included in the guidelines for FCR verification. This can lead to underdose of water. Secondly, several guidelines use turbidity (or cloudiness of the water) as a quantifiable indicator of water quality and use it for deciding the chlorine dose. Turbidity is an optical property of a suspension mainly attributed to the presence of suspended particles and can be measured with simple devices like a turbidity tube in the field [19]. Arguably, suspended matter may not be the best indicator of chemical properties of dissolved compounds in water. Gallandat et al. [20] suggest that kaolin clay-based turbidity has no impact on chlorine decay 
kinetics. Thus, it shows that the type of turbidity matters in terms of how it affects chlorine demand. Ali et al. [11] explored relationships between FCR decay and water quality in 220 unique samples and concluded that ambient air temperature and water conductivity show potential direct relationships with decay kinetics, while other water parameters including turbidity only accounted for about $25 \%$ of the variance in their data. To this end, parameters directly related to chlorine demand inducing compounds such as natural organic matter (NOM) (e.g., UV absorbance, dissolved or total organic carbon, etc.) could be a better metric to use [12]. Thirdly, several recommendations suggest verifying FCR at 30 min after dosing but do not consider chlorine decay for longer storage time [6,15]. Sometimes, it may be impractical to reach the recommended minimum FCR value of $0.2 \mathrm{mg} / \mathrm{L}$ for $24 \mathrm{~h}$ storage time [11]. Based on the assumption that households fetch their water daily, to ensure effective residual protection, dose recommendations should evaluate FCR decay during storage to up to $24 \mathrm{~h}$. Lastly, the values recommended by different guidelines vary, and it may create confusion for humanitarian staff in guideline selection.

To tackle the series of challenges present in determination of the adequate chlorine dose, a predictive tool, which uses the obtainable information in the field to rapidly and accurately estimate chlorine decay kinetics would be of use towards determining the appropriate initial dose for achieving safe free chlorination objectives. This paper examines several water parameters including NOM, water temperature, and 30-min chlorine demand and their impacts on chlorine decay kinetics. Model parameters from two of the chlorine decay kinetic models examined were found able to be calibrated with the above-mentioned water parameters. While chlorine decay models were typically calibrated with real chlorine decay data, the time constraint in the field limited the application of the models. Being able to calibrate model parameters with easily measurable water parameters allows the application of these models due to the substantial reduction in time requirement for calibration.

\section{Background}

\subsection{Two-Phase Chlorine Decay Behavior}

A typical chlorine decay curve (FCR versus time) contains two phases, a rapid decay phase takes place usually within the first 30 min after chlorine dosing and a slow decay phase afterwards [11,20,21]. Based on the chemistry of chlorine reactions, major contributors to the rapid decay phase are easily oxidizable substances, metals, fast reacting NOM, ammonia, etc. For the slow decay region, slow reacting NOM is considered the main reactant consuming chlorine, and the reaction rate is influenced by water temperature.

\subsection{Temperature}

The temperature dependence of chemical reaction rates can be described using the Arrhenius equation (Equation (1)) [12].

$$
k=A \times e^{-\frac{E_{a}}{R T}}
$$

where $A$ is the frequency term, meaning the frequency of collisions in the right orientation; $E_{a}$ is the activation energy of the reaction; $R$ is the universal gas constant; $k$ is the reaction rate, and $T$ is the temperature of the reaction in Kelvin. Powell et al. [22] has shown that an Arrhenius type relation exists between chlorine decay constants and the water temperature.

\subsection{Decay Kinetic Models}

There are a variety of kinetic models available in the literature for describing chlorine decay curves [23]. Wu [24] evaluated the application of seven basic chlorine decay models in humanitarian contexts. After excluding two models containing an additional stable term in the equation, which did not impact the model fitness significantly, five models were included as candidates in this study as shown in Table 2. They are Feben and Taras's empirical model, first order model, power model, 
parallel first order model and limited parallel power model. Later in the study, only Feben and Taras's empirical model and first order model were selected due to their simplicity and the high correlations between their decay term and the water parameters.

Table 2. Five Models evaluated by Wu (2020) [24] and their empirical solutions.

\begin{tabular}{|c|c|c|c|}
\hline Model & Equation ${ }^{1}$ & Data Modification OR Model Restrictions & References \\
\hline Feben and Taras's empirical model & $C=C_{0}-k t^{n}$ & Optionally fix $n$ value based on regression results & [25] \\
\hline First order model & $C=C_{0} \times e^{-k t}$ & Exclude data prior to $30 \mathrm{~min}$ reaction time & [26] \\
\hline Power model & $C=\left(k t \times(n-1)+\left(\frac{1}{C_{0}}\right)^{(n-1)}\right)^{\left(\frac{-1}{n-1}\right)}$ & Optionally fix $n$ value based on regression results & [26] \\
\hline Parallel first order model & $C=w \times C_{0} \times e^{-k_{1} t}+(1-w) \times C_{0} \times e^{-k_{2} t}$ & N/A & [26] \\
\hline Limited parallel power model & $\begin{array}{c}C=C^{*}+ \\
\left(k_{1} t \times\left(n_{1}-1\right)+\left(\frac{1}{(w)\left(C_{0}-C^{*}\right)}\right)^{\left(n_{1}-1\right)}\right)^{\left(\frac{-1}{n_{1}-1}\right)} \\
+\left(k_{2} t \times\left(n_{2}-1\right)+\left(\frac{1}{(1-w)\left(C_{0}-C^{*}\right)}\right)^{\left(n_{2}-1\right)}\right)^{\left(\frac{-1}{n_{2}-1}\right)}\end{array}$ & N/A & [27] \\
\hline
\end{tabular}

${ }^{1}$ For the listed equations, $C$ is the chlorine residual measured at time $t, C_{0}$ is the initial chlorine dose, $C^{*}$ is the stable component of chlorine added, $k$ is the decay constant term, $n$ is the power term, and $w$ is the ratio term.

For first order model, it is the most widely used chlorine decay model in conventional treatment system as it only has one parameter, ' $k$ ', to be calibrated [23]. Wu [24] suggested that first order model was potentially applicable to the humanitarian context when only data from the slow decay phase (30 min after dosing) were used as the model was unable to capture both phases at once.

\section{Materials and Methods}

The development of a chlorine dose predictive tool contained three main stages: (1) establishment of base relationships between the model parameters and selected water quality parameters using synthetic water (i.e., "brown bottle" tests), (2) verification of the base findings in scaled up systems (i.e., 20-L jerrycans), (3) verification of the models by conducting chlorine decay tests on water from three different natural sources.

To evaluate the relations between chlorine decay kinetics and the selected water quality parameters, synthetic water with different amounts of added humic acid (Sigma-Aldrich) and under different temperature settings were dosed with chlorine, and the decay of FCR were monitored over a duration of $24 \mathrm{~h}$. Selected models from Wu [24] were used to fit the decay data, and correlation analysis was conducted between the estimated decay constant term and water parameters, including organics (by measuring ultraviolet absorbance at wavelength of $\left.254 \mathrm{~nm}\left(\mathrm{UVA}_{254}\right)\right)$, temperature, and 30-min chlorine demand. A total of 64 chlorine decay tests, in both 125-mL glass brown bottles (Fisher Scientific, Canada) and 20-L plastic jerrycans (ULINE, Seattle, WA, USA) were conducted from August 2019 to January 2020.

\subsection{Apparatus Preparation}

The containers used in the tests were either $125-\mathrm{mL}$ brown bottles or 20-L jerrycans. The brown bottles were used for testing bulk chlorine decay, which means factors other than the characteristics of the tested water were minimized. The jerrycans were used to simulate chlorination in the field, and the results were compared to those obtained from bulk chlorine decay tests.

All glassware involved in the experiments were treated according to the Environmental Technology Verification Protocol before the test [28]. This cleaning protocol involves using Fl-70 detergent and chlorine solution with $10-20 \mathrm{mg} / \mathrm{L}$ available chlorine to remove the chlorine demand-inducing substances (i.e., adsorbed organics) on the inner surface of glassware.

\subsection{Synthetic Water Preparation}

Synthetic water was prepared in batches prior to testing. Humic acid (Sigma-Aldrich) was added to the Milli-Q water based on the relationship described by Equation (2) to achieve specific readings 
of $\mathrm{UVA}_{254}$, representing the amount of natural organic content in the water. This relationship was determined and used for the stock humid acid in the Public Health and Environmental Engineering (PH2E) laboratory at the University of Victoria (Victoria, Canada). The prepared water was then distributed into three 2-L Erlenmeyer flasks for temperature adjustments.

$$
Y=0.0281 \times X-0.0043
$$

where $Y$ is the water $\mathrm{UVA}_{254}$ value measured in $\mathrm{cm}^{-1}$, and $X$ is the concentration of humic Acid measured in $\mathrm{mg} / \mathrm{L}$.

\subsection{Temperature Control}

The test water was placed in three different incubators set at 10,20 , and $30^{\circ} \mathrm{C}$ respectively to reach the designed temperature. For tests in jerrycans, a water bath was set up to keep the water temperature at $30{ }^{\circ} \mathrm{C}$. Figure 1 illustrates the experimental setup of the tests.
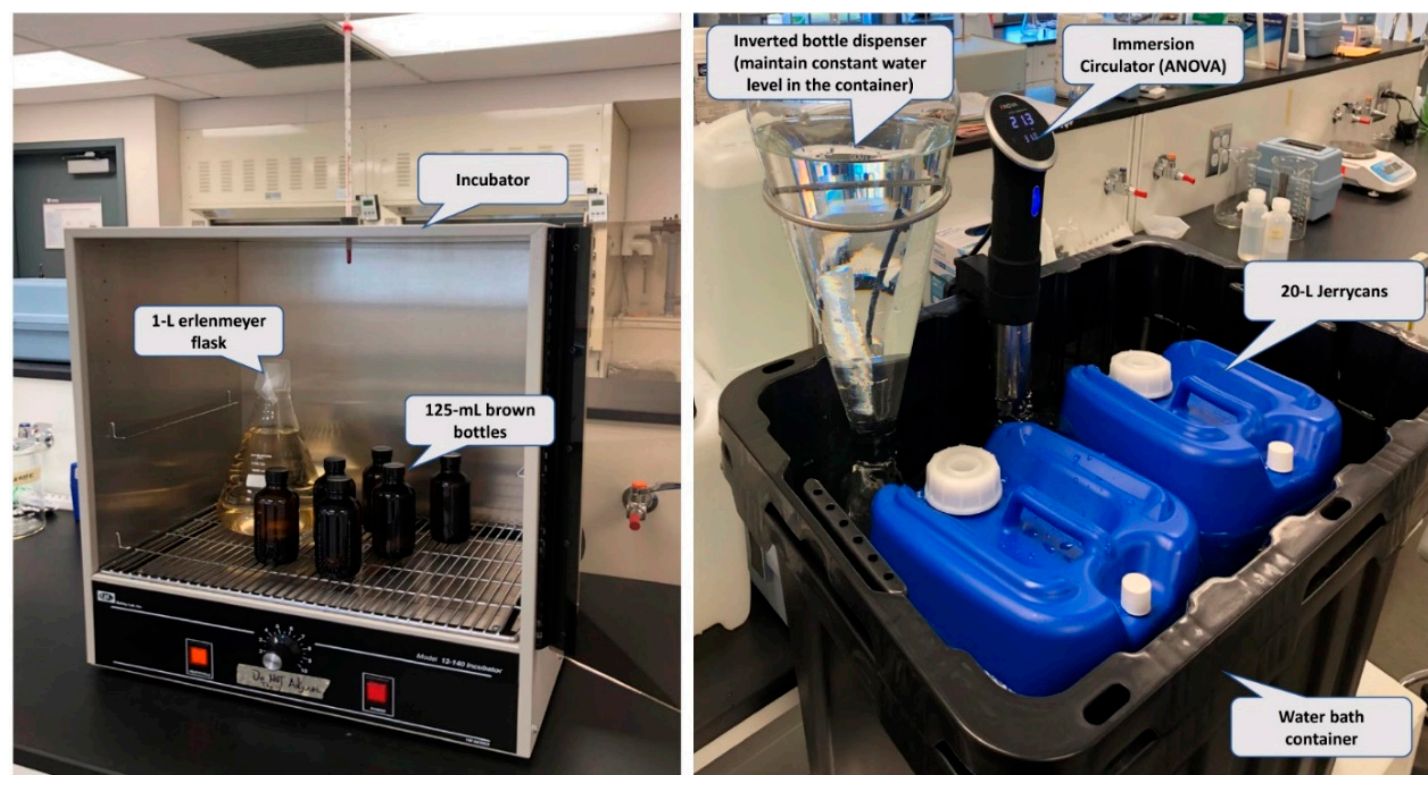

Figure 1. Experimental setup for chlorine decay tests in bulk "brown bottles" tests (left) and in jerrycans (right).

\subsection{Chlorine Stock Solution Preparation}

Chlorine stock solution was prepared by diluting commercially available bleach solution using Milli-Q water to reach a concentration of approximately $600 \mathrm{mg} / \mathrm{L}$ available chlorine. The strength of the stock chlorine solution was verified (HACH Method 8209) at least three times prior to each use. For each test, the stock solution was dosed to a separate container with Milli-Q water, parallel to the one with actual test water for dose verification.

\subsection{Matrix for Test Conditions}

The first stage of the study involves conducting a series of 24-h chlorine decay tests under various organics and temperature conditions. Figure 2 illustrates the matrix for the tested conditions inspired by the approach adopted by Gallandat et al. [20].

These 24-h decay tests were conducted in 125-mL brown bottles with synthetic water, incubated at the designed temperature. Chlorine residual readings were performed at 5, 10, 15, 20, 25, 30, 35, 40, and $45 \mathrm{~min}$ and $1,2,4,20$, and $24 \mathrm{~h}$ after dosing. These frequent readings increased the resolution of the chlorine decay curve, especially for the rapid decay phase in the first $30 \mathrm{~min}$. The second stage of 
the test was to conduct the same chlorine decay tests in 20-L jerrycans, which simulated the conditions of field water chlorination in a similar fashion to the setup used by Gallandat et al. [20]. The final stage of the test was to use water from three water sources, to verify the relationships developed in the first two stages.
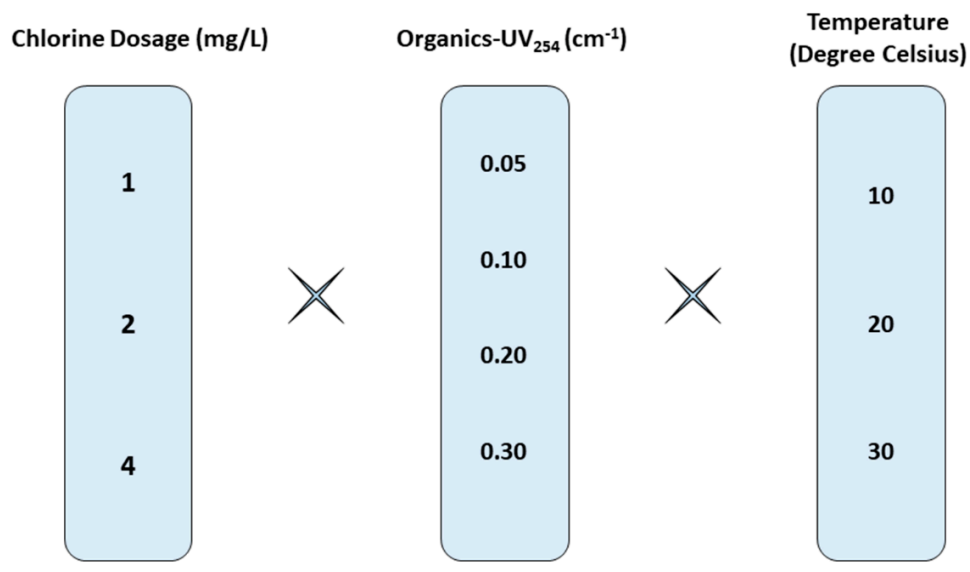

Figure 2. Matrix for the test conditions.

For chlorine dose selection, the $2 \mathrm{mg} / \mathrm{L}$ and $4 \mathrm{mg} / \mathrm{L}$ for clear and turbid water, respectively, were tested based on current recommendations $[8,28,29]$. Additionally, $1 \mathrm{mg} / \mathrm{L}$ was selected to examine a lower dose condition, which was a typical dose recorded in the South Sudan refugee camps [11].

For $\mathrm{UVA}_{254}$ settings, the selections were based on several studies, which determined the $\mathrm{UVA}_{254}$ value of numerous water sources (e.g., river and reservoir) ranging from 0.0 to $0.8 \mathrm{~cm}^{-1}$ [30-32], with the value for most cases found below $0.3 \mathrm{~cm}^{-1}$. Therefore, the selected values of $0.05,0.10,0.20$, $0.30 \mathrm{~cm}^{-1}$ represented most cases studied.

As for the temperature settings, 10,20 , and $30{ }^{\circ} \mathrm{C}$ were used to represent temperate climate conditions, room temperature conditions, and hot climate conditions, respectively. Even though specific values were selected to construct the test matrix, once the relationships between parameters were determined, any condition in between the extreme values tested could be interpolated and determined.

\subsection{Natural Water Samples Preparation}

Three water samples, representing three natural water types, were obtained for verifying the developed model. Water from an observation well located in the University of Victoria was used to represent groundwater. Water from the source reservoir, which supplies Victoria's drinking water, was selected to represent surface water. In addition, water from a pond located in a local golf course was tested to examine cases of more contaminated surface water. The sampled water was carried to the laboratory immediately and tested for turbidity, $\mathrm{pH}, \mathrm{UVA}_{254}$, and ammonia concentration. The water was then incubated and tested at $30^{\circ} \mathrm{C}$ for chlorine decay. All the water quality parameters were tested again before chlorine dosing.

\subsection{Data Analysis}

All three selected models were fitted to test data in MATLAB (R2019a) using the least squares method. Pearson's correlation analysis between parameters were conducted in Excel (2020) using its built-in Data Analysis toolbox.

\section{Results}

Overall, all models except first order model achieved satisfactory fitting on all 64 data sets, which were chlorine decay data in synthetic test water. The $\mathrm{R}^{2}$ distribution from data fitting for all five models are demonstrated in the box and whisker plot (Figure 3). Figure 4 shows three randomly 
selected representative tests from the experiments and the regression curves from the five models to demonstrate their fitness. As shown in the results, the different available chlorine decay models in literature, except for first order model, can describe chlorine decay behavior very well, with $100 \%$ of the regression achieved an $R^{2}$ value above 0.9 . The selection of models should be based primarily on model simplicity and the difficulty in model parameters calibration.

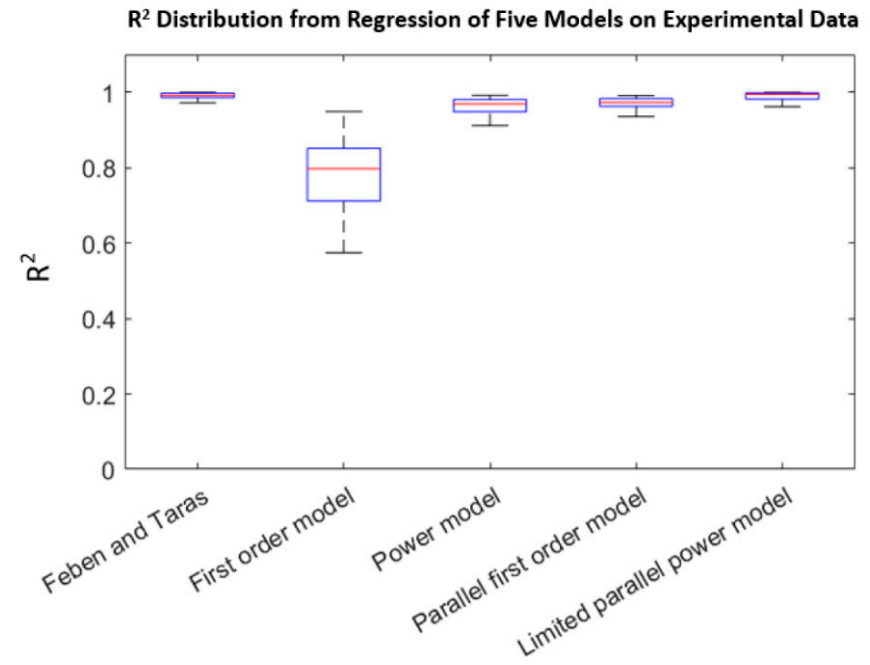

Figure 3. $\mathrm{R}^{2}$ from model fitting on all 64 chlorine decay tests using the five models. From top to bottom of each box are the maximum value, third quartile, median, first quartile, and minimum value of $R^{2}$ for the specific model it represents.

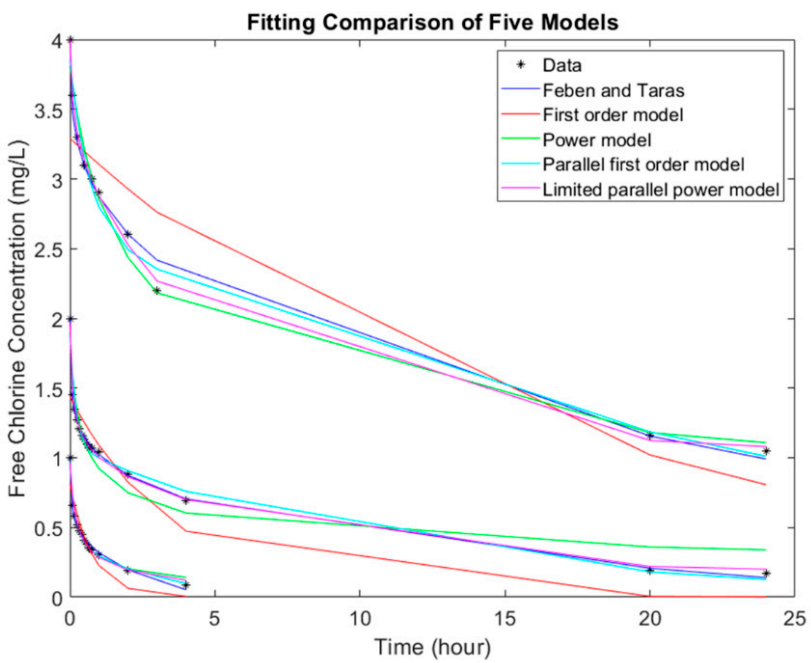

Figure 4. Model regression from the five models on three random tests from experiments.

\subsection{Correlation and Mathematical Relations}

Pearson's correlation analysis was conducted between estimated decay rate of the 36 bulk decay tests and their corresponding water quality parameters. The results from the analysis are shown in Table 3. Based on the results, $\mathrm{UVA}_{254}$ and 30-min chlorine demand of water appear to have significant impacts on chlorine decay rates. Feben and Taras's empirical model and first order model were selected for further analysis because of their simplicity and the higher correlation values obtained between their $k$ values and the water parameters. Due to the structures of these two models, water chemistry directly and solely impacts the $k$ value in the equation, leading to high correlations between $k$ and water parameters. For the rest of the models, the impacts from additional model parameters (i.e., $n$ in power models, $w$ in parallel models) likely lower the correlations between $\mathrm{k}$ and water parameters. 
Model parameters from both Feben and Taras's empirical model and first order model showed mathematical relationships with water's $U_{V A} 254$ value and the temperature (Figure 5). The decay constants for Feben and Taras's empirical model and first order model increased linearly and exponentially with the increase in $\mathrm{UVA}_{254}$, respectively (Figure 6). Both models' decay constants followed Arrhenius relationship with the water temperature, specifically, the linear relationship between the natural log of decay constant and the reciprocal of water temperature in Kelvin (Figure 6). When the water parameters were determined in the field, the ' $k$ ' value could be estimated based on the identified relations.

\section{Decay Constant vs. $\mathrm{UVA}_{254}$ and Temperature}
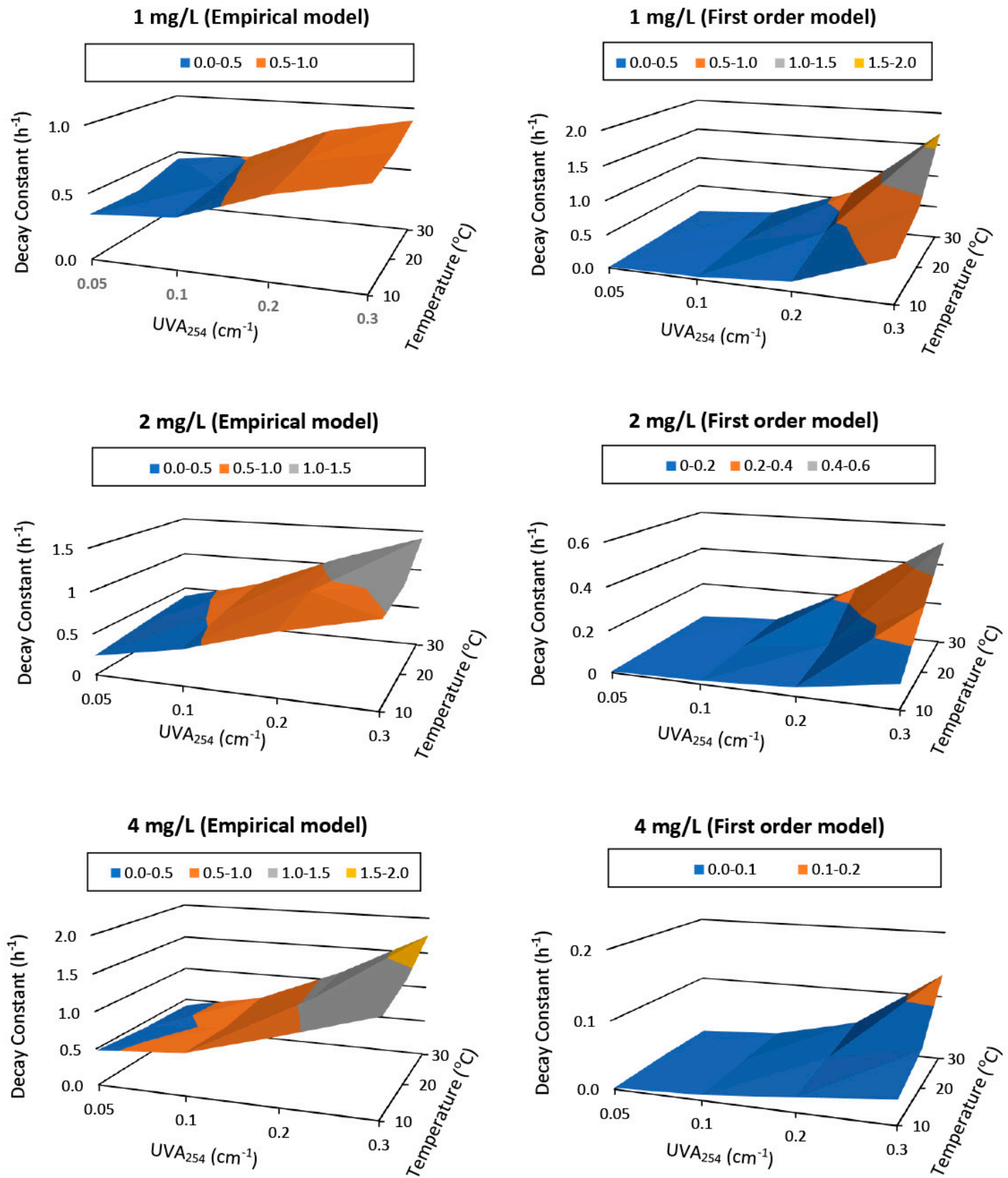

Figure 5. The combined impacts on chlorine decay constant in Feben and Taras's empirical model and first order model from temperature and natural organic matter (NOM) (all initial dose cases). 
Table 3. Pearson correlation results between chlorine decay term in the model and water parameters.

\begin{tabular}{ccccc}
\hline Models & Parameters & UVA $_{\text {254 }}$ & Temperature & 30-min Chlorine Demand \\
\hline Feben and Taras's empirical model & $k$ & 0.86 & 0.18 & 0.78 \\
First order model & $k$ & 0.67 & 0.24 & 0.77 \\
Power model & $k$ & 0.35 & 0.09 & 0.51 \\
Parallel first order model & $k_{1}$ & -0.32 & -0.19 & -0.28 \\
Parallel first order model & $k_{2}$ & 0.43 & 0.10 & 0.38 \\
Limited parallel power model & $k_{1}$ & 0.26 & -0.06 & 0.30 \\
Limited parallel power model & $k_{2}$ & 0.00 & -0.22 & -0.04 \\
\hline
\end{tabular}

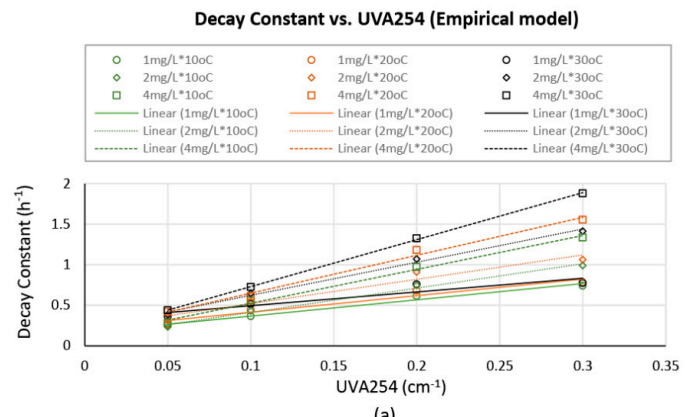

(a)

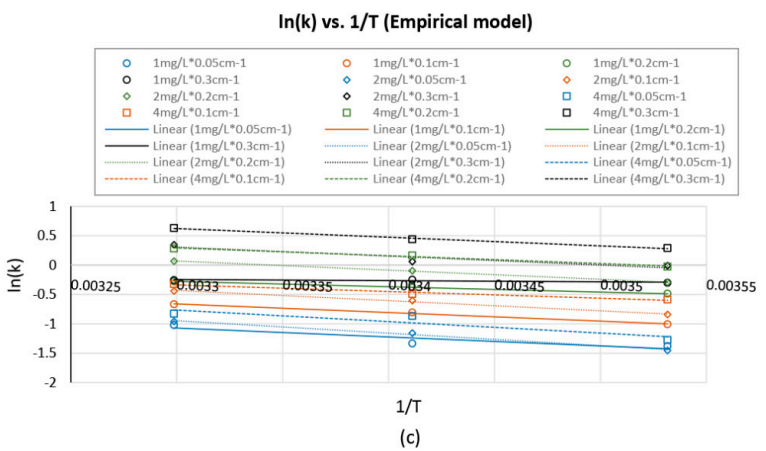

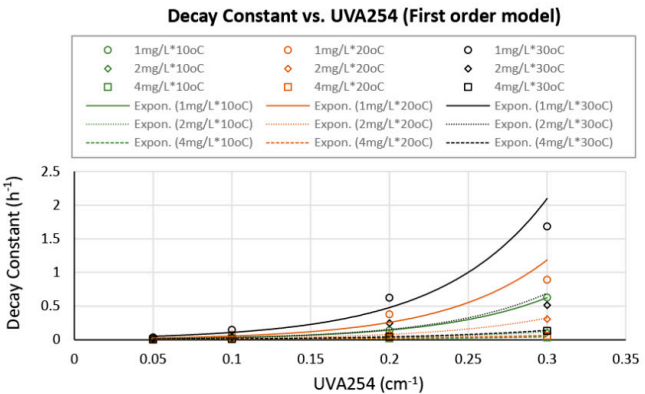

(b)

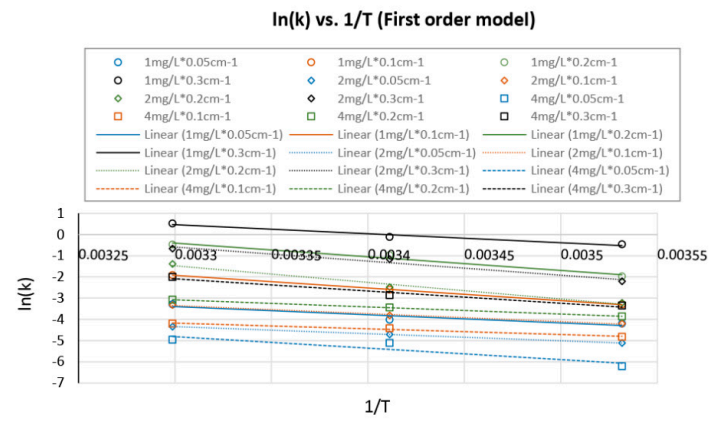

(d)

Figure 6. (a) Decay constants from Feben and Taras's empirical model versus NOM of the test water. (b) Decay constants from first order model versus NOM of the test water. (c) $\ln (k)$ versus $1 / T$ for all cases- Feben and Taras's empirical model. (d) $\ln (k)$ versus $1 / T$ for all cases-first order model. (Details can be found in Supplementary Material File S1).

\subsection{Decay Kinetics and Water Chlorine Demand}

The relations between decay constants estimated from data fitting and the 30-min chlorine demand of the tested water are shown in Figure 7. For Feben and Taras's empirical model and a specific initial dose, the decay constant followed linearly with the 30-min chlorine demand. For the first order model, since the fast decay phase (data before $30 \mathrm{~min}$ ) was excluded from the analysis, the initial dose changed from 1, 2, and $4 \mathrm{mg} / \mathrm{L}$ to the corresponding measured residual value at $30 \mathrm{~min}$ for each of the decay tests. Therefore, all the cases were plotted in the same figure. The relation between the two parameters was not obvious for all dose cases for first order model. However, generally, an exponential trend could be observed. 


\section{Decay constant versus 30-minute chlorine demand (Empirical model)}

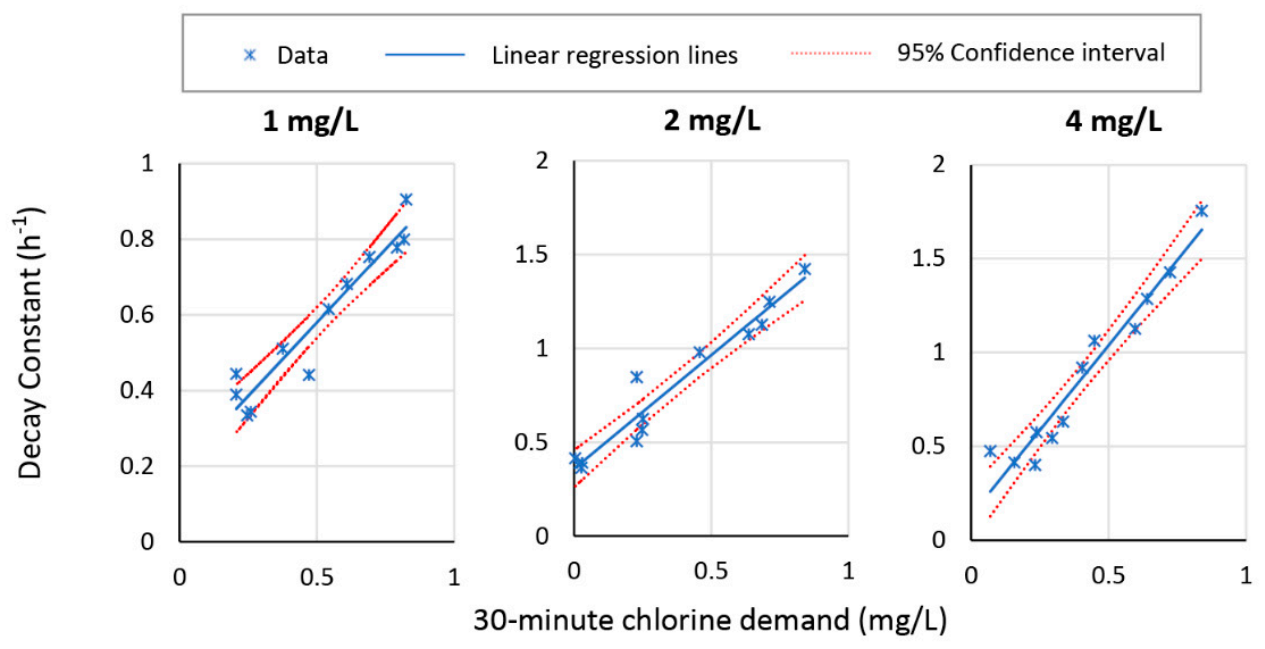

(a)

Decay constant versus 30-minute chlorine demand (First order model)

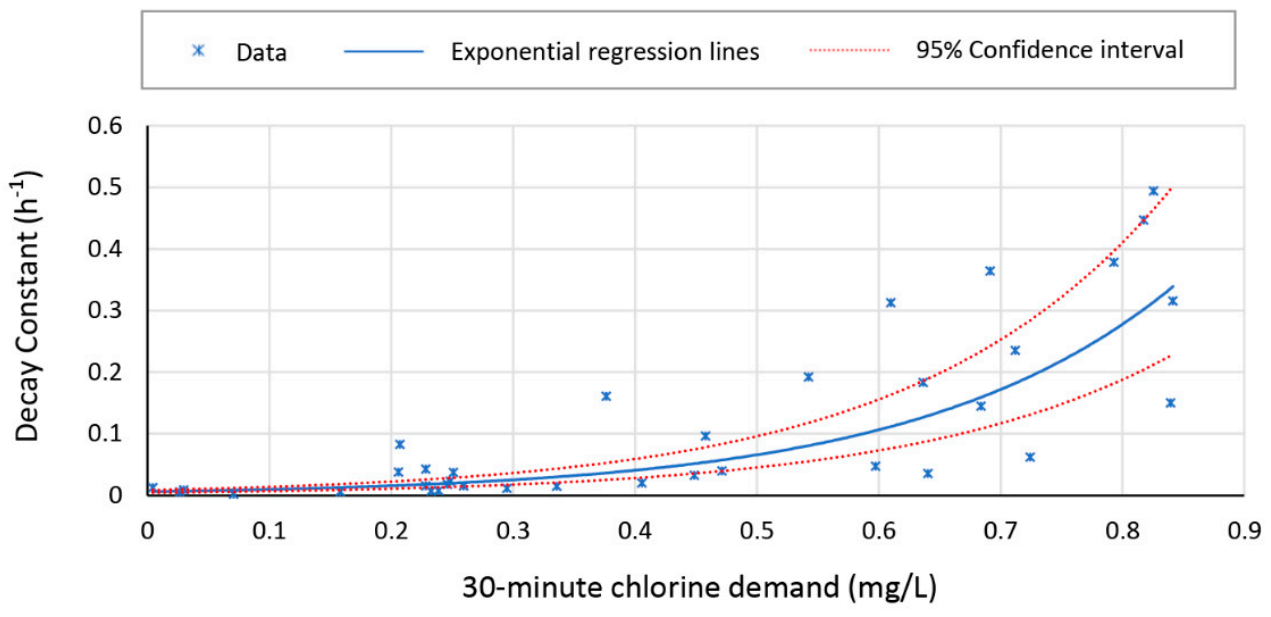

(b)

Figure 7. Mathematical relationship between decay constant and 30-min chlorine demand of test water. Figure (a) is for decay constant in Feben and Taras's empirical model. Figure (b) is for decay constant in first order model.

\subsection{Quality Control}

Four replicates were conducted for quality control purpose for the 36 bulk water tests. The four tests were selected randomly, and measured FCR were compared between the original and the replicates. Of the 36 FCR measurements in the four tests, the average error was $1 \%(-1 \%$ to $5 \%)$. For the eight replicates conducted for the 20-L jerrycans, the 96 measurements were all within $10 \%$ of the values from the initial tests.

\subsection{Verification}

Water quality data of the three natural water samples obtained in Victoria were summarized in Table 4 below. These values were measured right before the water was dosed with chlorine. The three water samples were dosed with a pre-determined dose of $4 \mathrm{mg} / \mathrm{L}$ chlorine, and the chlorine decay curves were predicted using the developed methods and compared with the actual measurements. A comparison of the predicted chlorine decay curves and actual decay curve can be seen in Figure 8 . 
Table 4. Water quality parameters of the three natural water sampled.

\begin{tabular}{cccccc}
\hline Water ID & Turbidity (NTU) & $\mathbf{p H}$ & $\mathbf{U V A}_{\mathbf{2 5 4}}\left(\mathbf{c m}^{-\mathbf{1}}\right)$ & Ammonia $(\mathbf{m g} / \mathbf{L})$ & 30-min Chlorine Demand $(\mathbf{m g} / \mathbf{L})$ \\
\hline Well Water & 12.6 & 7.52 & 0.009 & 0.01 & 0.11 \\
\hline Reservoir Water & 0.43 & 7.27 & 0.05 & 0.04 & 0.52 \\
\hline Pond Water & 9.67 & 6.3 & 0.212 & 0.43 & 3.12 \\
\hline
\end{tabular}

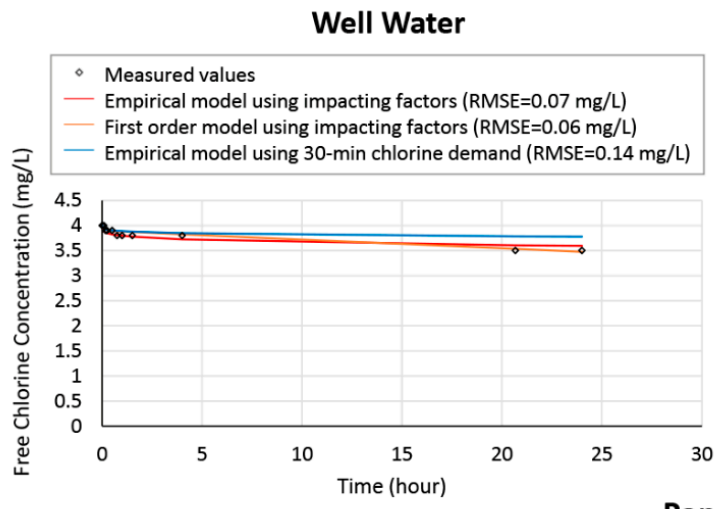

(a)

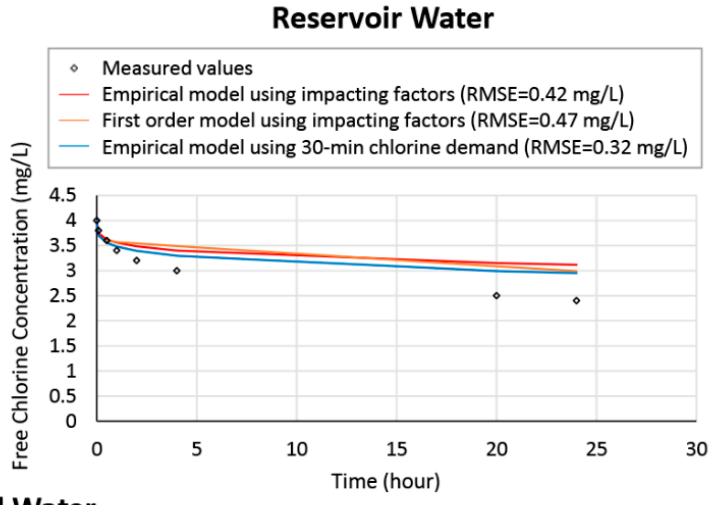

(b)

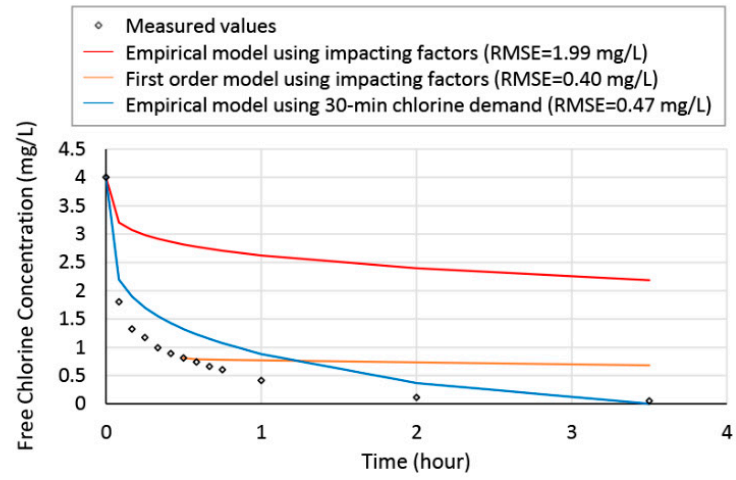

(c)

Figure 8. Predictions of chlorine decay in (a) well water, (b) reservoir water, and (c) pond water using the three developed models, and their comparisons with the measured values.

The two models with different methods for model parameter calibration performed similarly in prediction of chlroine decay for well water. Feben and Taras's empirical model with the decay constant calibrated based on water's 30-min chlorine demand could best capture the trend of chlorine decay with a relatively small root mean square error value for both reservoir water and pond water.

It can be seen in the case of well water that even though the turbidity of the water is higher (due to suspended mineral particles) than the other two water samples, the chlorine consumption is the lowest among the three water samples. This further proves that turbidity is a less ideal proxy for chlorine decay in comparison to $\mathrm{UVA}_{254}$ and 30-min chlorine demand.

\section{Discussion}

Traditionally, chlorine decay models need to be calibrated with extensive decay data for them to be able to describe chlorine decay in a water sample. This calibration requirement limits the application of chlorine decay models in contexts of humanitarian emergencies where data is usually unavailable and a rapid change of source water quality demands frequent model re-calibration.

This study showed that for simple model structures (Feben and Taras's empirical model and first order model), model parameters showed high correlations with water quality parameters. The two models explored in the study could be calibrated using either actual water quality parameters $\left(\mathrm{UVA}_{254}\right.$ and temperature) or using the 30-min chlorine demand, which is a proxy for the chlorine decay property 
of the water. This process substantially reduces the calibration requirement of the models, allowing the application of the chlorine decay models in humanitarian treatment contexts. The proposed model calibration methods also serve humanitarian field staff involved in water supply interventions by addressing the inadequacies and inconsistencies of current "rule-of-thumb" for chlorine dosing.

\subsection{Model Calibration Using Impacting Factors and Their Mathematical Relations}

Feben and Taras's empirical model contains two parameters, decay constant " $k$ " and power term " $n$ ", to be determined from regression analysis. To evaluate relations between decay constant " $k$ " and impacting factors, the influence from the power term " $n$ " needs to be minimized. After the initial model fitting, " $n$ " values generated from fitting the experimental data varied from 0.12 to 0.32 , with $50 \%$ of the values located between 0.2 and 0.26 . A second round of model fitting set the " $n$ " value fixed at 0.22 (the average value from the first round) to remove the influence from " $n$ ". As a result, the median $\mathrm{R}^{2}$ value decreased from 0.988 to 0.960 . The decay constant term was found linearly related to the change in water's organic content under all doses and temperature conditions but with different slope. The $\ln (k)$ over $1 / T$ plot (Figure 6 , plot c) showed linear relations between natural log of decay constant and the reciprocal of temperature in Kelvin. This verified the Arrhenius-type relationship. The slopes of the lines were very similar for all doses and organic content combinations with an average value of $1397 \mathrm{~K}$. Based on this result, the decay constant at a certain temperature from $10{ }^{\circ} \mathrm{C}$ to $30^{\circ} \mathrm{C}$ can be estimated according to Equation (3) when the decay constant is known for the same water at any known temperature from 10 to $30^{\circ} \mathrm{C}$.

$$
k_{1}=k_{2} \times e^{-1397 \times\left(\frac{1}{T_{1}}-\frac{1}{T_{2}}\right)}
$$

The analysis for the first order model was similar to those for the Feben and Taras's empirical model; the decay constant increased with the increase in water organic content and water temperature. The relationships between the $\mathrm{UVA}_{254}$ value and the decay constant under all three initial dosages conditions and all temperature settings were exponential. The four points for an initial dose of $1 \mathrm{mg} / \mathrm{L}$ in water with 0.2 and $0.3 \mathrm{~cm}^{-1}$ for the 20- and 30-degree curves in Figure 6 (plot b) did not follow the exponential trend, because the chlorine residual was completely reacted before $24 \mathrm{~h}$, and the decay kinetics for the entire period was not able to be calculated. This also meant that the initial chlorine was not sufficient to maintain a chlorine residual of greater than $0.2 \mathrm{mg} / \mathrm{L}$ for $24 \mathrm{~h}$ for the tested conditions. Otherwise, all chlorine decay kinetics increased exponentially with the increase in water organics content. For impacts from temperature, the $\ln (k)$ over $1 / T$ plot (Figure 6 , plot $d$ ) showed similar slopes of the regression lines for all doses and organic content combinations with an average value of $4818 \mathrm{~K}$. The values of the slope were less consistent in the case of first order model compared to those of Feben and Taras's empirical model. Equation (4) can be used to adjust the $k$ value for first order model based on the water's temperature.

$$
k_{1}=k_{2} \times e^{-4818 \times\left(\frac{1}{T_{1}}-\frac{1}{T_{2}}\right)}
$$

\subsection{Model Calibration Using 30-min Chlorine Demand}

It was not feasible to calibrate the first order model with $k$ using 30-min chlorine demand information because a large error was observed in the regression shown in Figure 7 (plot b). Additionally, since the trend was exponential, the decay constant could increase to an unrealistic level as the chlorine demand increased further. Calibrating Feben and Taras's empirical model with 30-min chlorine demand was feasible since the relations between estimated chlorine decay term and 30-min chlorine demand of water were linear. Feben and Taras's empirical model calibrated with water's 30-min chlorine demand produced the best prediction of chlorine decay in the natural water tested. This was likely due to the fact that 30-min chlorine demand incorporated other factors impacting chlorine decay, including temperature and chlorine consuming constituents. Thirty-min chlorine demand is also easily obtainable in the field. One disadvantage was that the relations between decay constant and measured 
30-min chlorine demand for different initial doses differed, which did not permit the chlorine decay rate estimation at intermittent doses. Currently, only relations for chlorine doses of 1, 2, and $4 \mathrm{mg} / \mathrm{L}$ were determined.

For achieving a minimum FCR of $0.2 \mathrm{mg} / \mathrm{L}$ at $24 \mathrm{~h}$ of storage time, the maximum decay constant term was determined for the three doses. Corresponding maximum 30-min chlorine demand of the water can be determined based on the identified relationships shown in Figure 7 (plot a). For an initial dose of 1,2 , and $4 \mathrm{mg} / \mathrm{L}$ chlorine, the 30 -min chlorine demand of the water cannot exceed $0.26,0.44$, and $0.97 \mathrm{mg} / \mathrm{L}$, respectively, to meet the FCR objective.

\subsection{Discussion on Discovered Relations}

Chlorine decay constant was observed to follow linear relationships with the $\mathrm{UVA}_{254}$ in the water. Humic acids mostly contain organics with aromatic compounds, and the dominate reactions between chlorine and the aromatic compounds are electrophilic substitution followed by stepwise substitutions [33]. An increase in humic acid concentration increases the available sites for the reactions. A linear increase in the $\mathrm{UVA}_{254}$ of water leads to linear increase in chlorine consumption demonstrated by the consumption term $k t^{n}$, in Feben and Taras's empirical model.

We hypothesized that humic substances and temperature were the two main factors impacting the slow decay phase, and the results from reservoir and pond water showed that there were more substances in the water leading to higher chlorine decay in the slow decay phase. Potential mechanisms include chlorine consumption from ammonia, chloramines, and other organics such as fulvic acids.

From tests in the study, the 24-h chlorine consumption ranged from $0.5 \mathrm{mg} / \mathrm{L}$ to $4 \mathrm{mg} / \mathrm{L}$ depending on the $\mathrm{UVA}_{254}$ values. However, the turbidity of all test water was below 10 NTU. This again suggested that turbidity was indeed not an adequate indicator for chlorine decay in test water in the study.

\subsection{Impacts from Storage Container}

For all 16 tests conducted in the jerrycans, the chlorine decay kinetics were similar to those from tests in brown bottles under the same organics and temperature conditions. This implies that chlorine reactions in (new/clean) jerrycans can be treated as bulk water decay. Within $24 \mathrm{~h}$, there were insignificant impacts from the environmental factors such as radiation and evaporation on chlorine decay. Additional chlorine reduction is possible in the field due to chlorine demand on the inner surface of the jerrycans. Frequent cleaning of the storage container can reduce chlorine demand from the containers and reduce the risk of water re-contamination [34].

\subsection{Additional Chlorine Reduction Mechanisms}

The organics and temperature-calibrated model underpredicted the decay rate of chlorine in surface water sources where additional chlorine consuming substances might be present. Either a coagulation/flocculation pre-treatment was needed before applying the predictive tool, or more chlorine reduction reactions need to be researched to expand the tool's applications.

\subsection{Outlook}

Ultimately, the discovered approach will be delivered through a user interface (e.g., spreadsheet and/or smartphone app). The models with their calibration methods can be built at the backstage. All the users need to do is to input the water parameters measured, and the tool automatically calculates and outputs the feasible initial doses for maintaining a $0.2 \mathrm{mg} / \mathrm{L}$ chlorine residual for $24 \mathrm{~h}$ of storage time. A preliminary tool (Supplementary Material File S2) is attached for demonstration.

Verification and refinement of the tool will need to be conducted in field test. The robustness of the tool can be verified in varied contexts, with different source water as well as in different water treatment/distribution methods. 


\section{Conclusions}

This study proves the possibility of chlorine decay model calibration using actual water parameters (UVA 254 , temperature, and 30-min chlorine demand) in the field, and this strategy satisfies the need for a practice-oriented, emergency-adapted dose prediction approach. With the discovered relations between chlorine decay model parameters and water parameters, humanitarian field staff can determine the appropriate initial chlorine dose for achieving FCR objectives by measuring several obtainable field water quality parameters.

Thirty-min chlorine demand was shown able to reflect actual chlorine decay kinetics. Together with the fact that it can be easily obtained in the field, it is recommended that 30-min chlorine demand be used as an indicator for chlorine dose design instead of using turbidity. Based on the relations discovered in the study, for an initial dose of 1,2, and $4 \mathrm{mg} / \mathrm{L}$ chlorine, the 30-min chlorine demand of the water cannot exceed $0.26,0.44$, and $0.97 \mathrm{mg} / \mathrm{L}$, respectively, for achieving a minimum FCR of $0.2 \mathrm{mg} / \mathrm{L}$ at $24 \mathrm{~h}$ of storage. The 30-min chlorine demand incorporates impacts such as $\mathrm{UVA}_{254}$ and temperature on chlorine decay kinetics. The exact values of the parameters were not required to be determined. Only 30-min chlorine demand is needed to evaluate if the initial doses were feasible for the test water in field.

To develop the tool for finding the appropriate initial dose, Feben and Taras's empirical model can be used as the basic model. The power term " $n$ " in the equation can be set fixed at 0.22 , and the decay term " $\mathrm{k}$ " can be estimated with an embedded formula based on the three mathematical relations developed for the three initial doses in the study. The three doses, 1, 2, and $4 \mathrm{mg} / \mathrm{L}$, can be evaluated at once on whether they are able to reach $0.2 \mathrm{mg} / \mathrm{L}$ FCR at $24 \mathrm{~h}$ based on the input value of 30-min chlorine demand. Doses able to achieve the objective are output for the humanitarian staff.

Supplementary Materials: The following are available online at http://www.mdpi.com/2073-4441/12/5/1506/s1, File S1: Parameter Relations, File S2: Chlorine Dose Evaluation Tool.

Author Contributions: Conceptualization, C.C.D.; methodology, C.C.D. and H.W.; software, H.W.; validation, H.W.; formal analysis, H.W.; investigation, H.W.; resources, C.C.D.; data curation, H.W.; writing-original draft preparation, H.W.; writing-review and editing, C.C.D. and H.W.; visualization, H.W.; supervision, C.C.D.; project administration, C.C.D.; funding acquisition, C.C.D. All authors have read and agreed to the published version of the manuscript.

Funding: This research was funded by the Humanitarian Innovative Fund (HIF) and the Natural Sciences and Engineering Research Council of Canada (NSERC).

Acknowledgments: We would like to thank Capital Regional District (CRD) for providing the reservoir water used in this study. We would also like to thank Arielle Garrett for the assistance in the laboratory.

Conflicts of Interest: The authors declare no conflict of interest. The funders had no role in the design of the study; in the collection, analyses, or interpretation of data; in the writing of the manuscript; or in the decision to publish the results.

\section{References}

1. Davis, J.; Lambert, R. Engineering in Emergencies: A Practical Guide for Relief Workers; ITDG: London, UK, 2002.

2. Sphere Project. The Sphere Handbook: Humanitarian Charter and Minimum Standards in Humanitarian Response, 2018 ed.; Sphere Association: Geneva, Switzerland, 2018; Available online: https://spherestandards.org/wpcontent/uploads/Sphere-Handbook-2018-EN.pdf (accessed on 2 March 2020).

3. Dorea, C. Comment on "Emergency water supply: A review of potential technologies and selection criteria". Water Res. 2012, 46, 6175-6176. [CrossRef] [PubMed]

4. Dorea, C.; Bertrand, S.; Clarke, B. Particle separation options for emergency water treatment. Water Sci. Technol. 2006, 53, 253-260. [CrossRef] [PubMed]

5. Lantagne, D.; Yates, T. Household Water Treatment and Cholera Control. J. Infect. Dis. 2018, 218, S147-S153. [CrossRef] [PubMed]

6. World Health Organization (WHO). Household Water Treatment and Safe Storage Following Emergencies and Disasters; WHO: Geneva, Switzerland, 2005. 
7. Branz, A.; Levine, M.; Lehmann, L.; Bastable, A.; Ali, S.I.; Kadir, K.; Yates, T.; Bloom, D.E.; Lantagne, D. Chlorination of drinking water in emergencies: A review of knowledge to develop recommendations for implementation and research needed. Waterlines 2017, 36, 4-39. [CrossRef]

8. Murray, A.L.; Lantagne, D. Accuracy, precision, usability, and cost of free chlorine residual testing methods. J. Water Health 2014, 13, 79-90. [CrossRef]

9. Lantagne, D.S. Sodium hypochlorite dosage for household and emergency water treatment. J.-Am. Water Work. Assoc. 2008, 100, 106-119. [CrossRef]

10. Abdel-Gawad, S.T.; Bewtra, J.K. Decay of chlorine in diluted municipal effluents. Can. J. Civ. Eng. 1988, 15, 948-954. [CrossRef]

11. Ali, S.I.; Ali, S.S.; Fesselet, J.-F. Effectiveness of emergency water treatment practices in refugee camps in South Sudan. Bull. World Health Organ. 2015, 93, 550-558. [CrossRef]

12. Crittenden, J.C.; Trussell, R.R.; Hand, D.W.; Howe, K.J.; Tchobanoglous, G. MWH's Water Treatment: Principles and Design; Wiley: Hoboken, NJ, USA, 2012.

13. Monteiro, L.; Viegas, R.; Covas, D.; Menaia, J. Modelling chlorine residual decay as influenced by temperature. Water Environ. J. 2015, 29, 331-337. [CrossRef]

14. Centers for Disease Control and Prevention (CDC). Making Water Safe in an Emergency; CDC: Atlanta, GA, USA, 2019. Available online: https://www.cdc.gov/healthywater/emergency/drinking/making-water-safe.html (accessed on 2 March 2020).

15. United States Environmental Protection Agency (US EPA). Emergency Disinfection of Drinking Water; US EPA: Washington, DC, USA, 2017; p. 2. Available online: https://www.epa.gov/sites/production/files/2017-09/ documents/emergency_disinfection_of_drinking_water_sept2017.pdf (accessed on 2 March 2020).

16. Johns Hopkins University (JHU); International Federation of Red Cross and Red Crescent Societies (IFRC). Public Health Guide in Emergencies, 2nd ed.; Johns Hopkins University (JHU): Baltimore, MD, USA; International Federation of Red Cross and Red Crescent Societies (IFRC): Geneva, Switzerland, 2008.

17. Federal Ministry of Health (FMH). Protocols for the Chlorination of Drinking Water (for Small to Medium Sized Supplies); Government of Sudan: Khartoum, Sudan, 2017.

18. Nicoletti, M.A.; Siqueira, E.L.; Bombana, A.C.; De Oliveira, G.G. Shelf-life of a $2.5 \%$ sodium hypochlorite solution as determined by Arrhenius equation. Braz. Dent. J. 2009, 20, 27-31. [CrossRef]

19. Dorea, C.; Simpson, M.R. Turbidity tubes for drinking water quality assessments. J. Water Sanit. Hyg. Dev. 2011, 1, 233-241. [CrossRef]

20. Gallandat, K.; Stack, D.; String, G.M.; Lantagne, D. Residual Maintenance Using Sodium Hypochlorite, Sodium Dichloroisocyanurate, and Chlorine Dioxide in Laboratory Waters of Varying Turbidity. Water 2019, 11, 1309. [CrossRef]

21. Al Heboos, S.; Licskó, I. Application and Comparison of Two Chlorine Decay Models for Predicting Bulk Chlorine Residuals. Period. Polytech. Civ. Eng. 2016, 61, 7-13. [CrossRef]

22. Powell, J.C.; Hallam, N.B.; West, J.R.; Forster, C.F.; Simms, J. Factors which control bulk chlorine decay rates. Water Res. 2000, 34, 117-126. [CrossRef]

23. Fisher, I.; Kastl, G.; Sathasivan, A. Evaluation of suitable chlorine bulk-decay models for water distribution systems. Water Res. 2011, 45, 4896-4908. [CrossRef]

24. Wu, H. Achieving Safe Free Residual Chlorination at Point-of-Use in Emergencies: A Modellling Approach; University of Victoria: Victoria, BC, Canada, 2020.

25. Feben, D.; Taras, M.J. Studies on Chlorine Demand Constants. J.-Am. Water Work. Assoc. 1951, 43, 922-931. [CrossRef]

26. Haas, C.N.; Karra, S.B. Kinetics of wastewater chlorine demand exertion. J. Water Pollut. Control Fed. 1984, $56,170-173$.

27. Kim, H.; Koo, J.; Kim, S. A general framework of chlorine decay modeling at a pilot-scale water distribution system. J. Water Supply Res. Technol. 2014, 64, 543-557. [CrossRef]

28. United States Environmental Protection Agency (US EPA). Protocol for Equipment Verification Testing for Removal of Precursors to Disinfection ByProducts; Diane Publishing: Darby, PA, USA, 2003.

29. World Health Organization (WHO). Guidelines for Drinking-Water Quality; WHO: Geneva, Switzerland, 2017.

30. Bond, T.; Goslan, E.H.; Parsons, S.; Jefferson, B. Treatment of disinfection by-product precursors. Environ. Technol. 2011, 32, 1-25. [CrossRef] 
31. Edzwald, J.K.; Becker, W.C.; Wattier, K.L. Surrogate Parameters for Monitoring Organic Matter and THM Precursors. J.-Am. Water Work. Assoc. 1985, 77, 122-132. [CrossRef]

32. Volk, C.; Wood, L.; Johnson, B.; Robinson, J.; Zhu, H.W.; Kaplan, L. Monitoring dissolved organic carbon in surface and drinking waters. J. Environ. Monit. 2002, 4, 43-47. [CrossRef] [PubMed]

33. Deborde, M.; Von Gunten, U. Reactions of chlorine with inorganic and organic compounds during water treatment-Kinetics and mechanisms: A critical review. Water Res. 2008, 42, 13-51. [CrossRef] [PubMed]

34. Meierhofer, R.; Wietlisbach, B.; Matiko, C. Influence of container cleanliness, container disinfection with chlorine, and container handling on recontamination of water collected from a water kiosk in a Kenyan slum. J. Water Health 2019, 17, 308-317. [CrossRef] [PubMed]

(C) 2020 by the authors. Licensee MDPI, Basel, Switzerland. This article is an open access article distributed under the terms and conditions of the Creative Commons Attribution (CC BY) license (http://creativecommons.org/licenses/by/4.0/). 